\title{
Mortalidad evitable y políticas en salud. Colombia, 1985-2002
}

\author{
Rubén Darío Gómez-Arias, PhD' 1 , Andreu Nolasco Bonmati, PhD², \\ Pamela Pereyra-Zamora, MS ${ }^{3}$, Fabio León Rodríguez-Ospina, MSc ${ }^{4}$, \\ Sandra Milena Agudelo-Londoño, GSIS ${ }^{5}$
}

\section{RESUMEN}

Introducción: La confianza en la capacidad de evitar algunas muertes o diferir su aparición es el fundamento de toda política de salud, uno de cuyos principales resultados debe ser reducir las muertes evitables, y controlar las condiciones que aumentan el riesgo de morir.

Objetivos: Establecer variaciones en la tendencia de la mortalidad evitable(ME) registrada en Colombia entre 1985 y 2002, como indicadoras del impacto efectivo que las reformas en la política sanitaria pudieran haber tenido sobre sus determinantes.

Métodos: Estudio de la ME con base en los registros oficiales de defunción y en las proyecciones censales de Colombia entre 1985-2002. Para determinar la evitabilidad, se aplicó un inventario de causas de ME (ICME) ajustado a las condiciones epidemiológicas del país durante el período que se analiza.

Resultados: De las muertes registradas, $75.3 \%$ se clasificaron como evitables. Se identificaron siete patrones de tendencia que reflejan, de manera particular, los efectos de las políticas públicas sobre los determinantes de la mortalidad.

Conclusiones: En general, la ME viene disminuyendo en Colombia desde 1985 en la población general y entre los hombres, sin variaciones significativas durante el período. Las variaciones en la tendencia de las tasas ajustadas de varios grupos de causas hacen pensar en un deterioro en el control de sus determinantes, especialmente desde 1990. Los cambios aplicados en las políticas públicas durante los últimos años no se reflejaron en un mejor control de las muertes evitables, aunque el gasto en salud aumentó de modo muy notable en el país.

Palabras clave: Mortalidad evitable; Mortalidad; Colombia; Política de salud; Reformas sanitarias.

Avoidable mortality and health policies. Colombia. 1985-2002

\section{SUMMARY}

Introduction: Confidence in the capacity to avoid some deaths or to defer their occurrence is the foundation of any health public policy, one of whose main results should be to reduce avoidable mortality by controlling conditions that increase the risk of dying.

Objectives: To establish trend variations in avoidable mortality (AM), registered in Colombia between 1985 and 2002, as an indicator of the effective impact that recent health reforms could have produced on the determinants of mortality.

Methods: This is a study of AM, based on Colombian official registries of death and census projections, between 1985 and 2002. To determine the avoidability, an inventory of causes of AM (ICAM), based on the Holland and Taucher models, was applied to the data during the period analyzed.

Results: Of the number of deaths registered, $75.3 \%$ were classified as avoidable. Seven tendency patterns were identified and each of them reflects, in particular, public-policy effects on mortality determinants.

1. Profesor Titular, Facultad Nacional de Salud Pública, Universidad de Antioquia, Medellín, Colombia.

e-mail:rdgomez@guajiros.udea.edu.co

2. Catedrático, escuela Universidad de Alicante, Unidad de Investigación de Análisis de la mortalidad y Estadísticas Sanitarias, Universidad de Alicante, España. e-mail: nolasco@ua.es

3. Unidad de Investigación de Análisis de la Mortalidad y Estadísticas Sanitarias, Universidad de Alicante, España. e-mail:pamela.pereyra@ua.es

4. Profesor, Facultad Nacional de Salud Pública, Universidad de Antioquia, Medellín, Colombia. e-mail: flrodri@guajiros.udea.edu.co

5. Joven investigadora, Gerente de Sistemas de Información en Salud, Facultad Nacional de Salud Pública, Universidad de Antioquia, Medellín, Colombia. e-mail: sandraagudelo@guajiros.udea.edu.co

Recibido para publicación julio 24, 2008 Aceptado para publicación septiembre 30, 2009 
Conclusions: On the whole, AM has diminished in Colombia since 1985 among the general population and among men, without significant variations during the period. The trend variations observed of adjusted rates suggest deterioration in the control of AM determinants, particularly since 1990. Public policy changes applied in Colombia during the period have not been reflected in a better control of avoidable deaths, although health expenses have increased remarkably.

\section{Keywords: Avoidable death; Mortality; Colombia; Health policy; Health reform.}

El informe Salud Mundial 2000 de la Organización Mundial de la Salud (OMS) ha generado un importante debate sobre las políticas públicas que afectan la salud y los criterios para su evaluación ${ }^{1,2}$. La idea de utilizar evidencias para evaluar políticas públicas se ha abierto camino desde mediados de 1980, y en este campo los análisis de la mortalidad evitable ${ }^{3}(\mathrm{ME})$ constituyenuno de los recursos disponibles más importantes. De acuerdo con Rutstein et al. ${ }^{4}$ una muerte se considera evitable, si todo lo que se debía hacer se hubiera hecho, por tanto, tal defunción se hubiera prevenido o retrasado. Adicionalmente Holland et al..$^{5}$ propusieron que para aceptar la evitabilidad de una muerte debe identificarse un tipo particular de intervención considerada potencialmente efectiva para prevenirla o postergarla. Desde un enfoque distinto, Uemura\& $\mathrm{Pisa}^{6}$ argumentan que las tasas mínimas de mortalidad logradas por una población reflejan su capacidad para evitar la muerte hasta ese punto, y que si los recursos estuvieran equitativamente repartidos, todas las poblaciones deberían tener tasas similares; en consecuencia, las muertes que una sociedad ha logrado evitar debieran considerarse también evitables en las demás, y las diferencias observadas entre ellas son una manifestación de inequidad. Los enfoques de la ME reflejan la concepción epistemológica, el interés y la disponibilidad de recursos al alcance de los analistas; algunos de ellos centran la evitabilidad en las acciones de atención médica ${ }^{7}$; otros, en cambio, atribuyen la mortalidad a condiciones biológicas, económicas, ambientales y culturales que interactúan para producir la muerte, algunas de las cuales se pueden controlar con la tecnología disponible. En todos los casos se considera que la condición de «evitabilidad» (avoidability) no es una propiedad natural de la muerte sino una condición resultante de múltiples factores que confluyen en el desenlace final ${ }^{3,8}$. En tal sentido, la ME constituye una fuente de indicadores de desempeño de políticas dirigidas a controlar los determinantes del proceso saludenfermedad. Desde 1980, Colombia ha puesto en marcha reformas políticas en el ámbito económico y social que han modificado sustancialmente la gestión del Estado y de los particulares sobre los determinantes de la salud. En 1990, la Ley 10 desmontó el sistema nacional de salud, SNS, que operaba desde 1975 yen 1993 la Ley 100 creó el sistema general de seguridad social en salud (SGSSS) que reorganizó los servicios de salud con criterios de mercado. Las reformas colombianas fueron, durante la década de 1990, defendidas por algunos analistas como el modelo más promisorio de reforma sanitaria del tercer mundo ${ }^{1}$ y atacada por otros como un retroceso en relación con la equidad, el bienestar colectivo y el derecho a la salud ${ }^{9}$. La reforma de 1993 sustituyó un modelo estatal de prestación de servicios que se financian con subsidios destinados a cubrir la oferta de los hospitales públicos, por un modelo de competencia regulada con base en el mercado del aseguramiento, donde múltiples agentes estatales y privados ofrecen los servicios de saludse, de conformidad con el tipo de seguro que pueda pagar el usuario. La reforma incluyó un importante incremento en los recursos de salud; las cifras difieren según los estudios ${ }^{10}$ pero ciertos análisis conservadores sugieren que el gasto público total en salud pasó de $1.4 \%$ del PIB en 1993 a $3.1 \%$ en $2003^{11}$. Los costos económicos y sociales de la reforma, y las implicaciones potenciales de su aplicación sobre diversas dimensiones del bienestar colectivo de los colombianos, exigen una evaluación exhaustiva que dé cuenta de su complejidad, y muy especialmente de sus efectos sobre las enfermedades y defunciones evitables, cuyo control debe ser uno de los objetivos centrales de las políticas públicas. Al asumir que la efectividad de los cambios generados por las reformas sanitarias debía reflejarse de alguna manera en las defunciones, el estudio se propuso descubrir variaciones en la estructura y la tendencia de las muertes registradas en Colombia entre 1985 y 2002 , que pudieran sugerir el éxito o el fracaso de tales políticas sobre los determinantes de la mortalidad.

\section{MÉTODOS}

Se realizó un análisis de la mortalidad basado en las proyecciones censales y en la base de datos de los 
registros oficiales de defunción provistos por el Departamento Nacional de Estadística (DANE) de Colombia, entidad responsable de proporcionar estos informes. La población de referencia estuvo constituida por todas las defunciones registradas oficialmente en el país en el período 1985-2002, que se codificaron de acuerdo con la Clasificación Internacional de Enfermedades $9^{\text {a }}$ Revisión (CIE-9) para el período 1985-1996, y con la $10^{\mathrm{a}}$ Revisión (CIE-10)12 para 1997-2002. Ambas clasificaciones se homologaron a la Lista Condensada 1 de 103 causas propuesta por la OMS ${ }^{12}$.

Se considera que el registro de la mortalidad ha venido en mejoría desde 1980, en especial en las grandes ciudades; sin embargo la omisión es aún alta y para 2005 se estimaba en $9.3 \%$, aunque en algunas regiones podría ser mayor ${ }^{13}$. Durante el período de estudio el sistema de registro de la mortalidad experimentó dos modificaciones importantes: a partir de 1997 la $C I E-10$ sustituyó a la $C I E-9$, y en 1998 al formulario de registro de la mortalidad se hicieron ajustes al relacionados sobre todo con las muertes obstétricas, la mortalidad perinatal y las muertes hospitalarias. Las defunciones registradas en la base de datos se clasificaron con fundamento en un inventario de causas de ME (ICME) basado en los criterios de Holland ${ }^{7}$ y de Taucher $^{14}$ y según el perfil epidemiológico de Colombia ${ }^{8,15}$; al ajustar el instrumento se estableció que sus mediciones superan a las obtenidas con el listado de Taucher en un $25.4 \%$, y a la obtenida con el listado de Holland en un 58.5\%; las diferencias obedecen a la incorporación de causas de muerte comunes en Colombia que no se reconocen como evitables por estos listados, entre ellas homicidios (que sí es reconocida como evitable por Taucher), malaria, fiebre amarilla y anemia ${ }^{8}$.

El ICME adoptó las cuatro categorías de Taucher para agrupar las causas evitables ${ }^{14}$ pues considera que reflejan un enfoque más integral de las intervenciones relacionadas con la evitabilidad, y que sus criterios pretenden dar cabida a acciones específicas de prevención primaria (grupo A), a intervenciones médicas sobre el individuo (grupo B), a acciones sobre el ambiente (grupo C) y a medidas mixtas más complejas realizadas sobre el sujeto, el entorno físico y la sociedad (grupo D). Las demás causas de muerte se agruparon en tres categorías:

a) Causas no evitables (CNE).

b) Mortalidad por causas mal definidas (MCM) que incluye Sintomas, signos y hallazgos no descritos en otra parte.

c) Otras causas de muerte de difícil clasificación (MOC), categoría conformada predominantemente por el resto de causas del sistema respiratorio, del sistema digestivo y del sistema nervioso central.

Para cada año se obtuvieron los recuentos, las tasas brutas y ajustadas y la razón comparativa de mortalidad (RCM). Las tasas anuales se calcularon por edad y sexo y se ajustaron por el método directo contra la población estándar de OMS-OPS. Como denominadores se siguieron las proyecciones censales publicadas oficialmente por el DANE; a este respecto, los estudios de conciliación censal consideran que las proyecciones de los últimos años han sobreestimado la población real en cerca de $7.5 \%{ }^{13}$.

La RCM se calculó por el método directo sobre tasas ajustadas, utilizando como estándar el riesgo observado en el mismo subgrupo en el último año analizado; los intervalos de confianza de la RCM se calcularon asumiendo para las tasas una distribución de Poisson y la aplicación estadística Poisson regression del Programa Egret ${ }^{\circledR}$. Conla intención de identificary seleccionar puntos donde hubiese habido cambios significativos en la tendencia de las tasas y establecer su ritmo anual de variación, se hicieron análisis de joinpoint. La regresión de joinpoint es un procedimiento estadístico de regresión parcial, que modela los datos, descubre puntos de inflexión ${ }^{16,17}$ y permite probar si un cambio aparente en la tendencia de una variable es o no estadísticamente significativo. Para los análisis de joinpoint se utilizó el software Joinpoint del Surveillance Research Program del US National Cancer Institute, versión 3.0; los análisis se realizaron en las condiciones predefinidas por el software, que excluyen la localización de puntos de inflexión a menos de dos observaciones del siguiente punto de quiebre o de los extremos de la serie. El estudio contó con la aprobación del Comité de Ética de la Facultad Nacional de Salud Pública.

\section{RESULTADOS}

El Cuadro 1 muestra la estructura de la mortalidad registrada en el país durante los 18 años analizados; entre los varones predominaron las afecciones cardiovasculares, las muertes por causas externas (homicidios y accidentes) y los tumores; entre las mujeres 


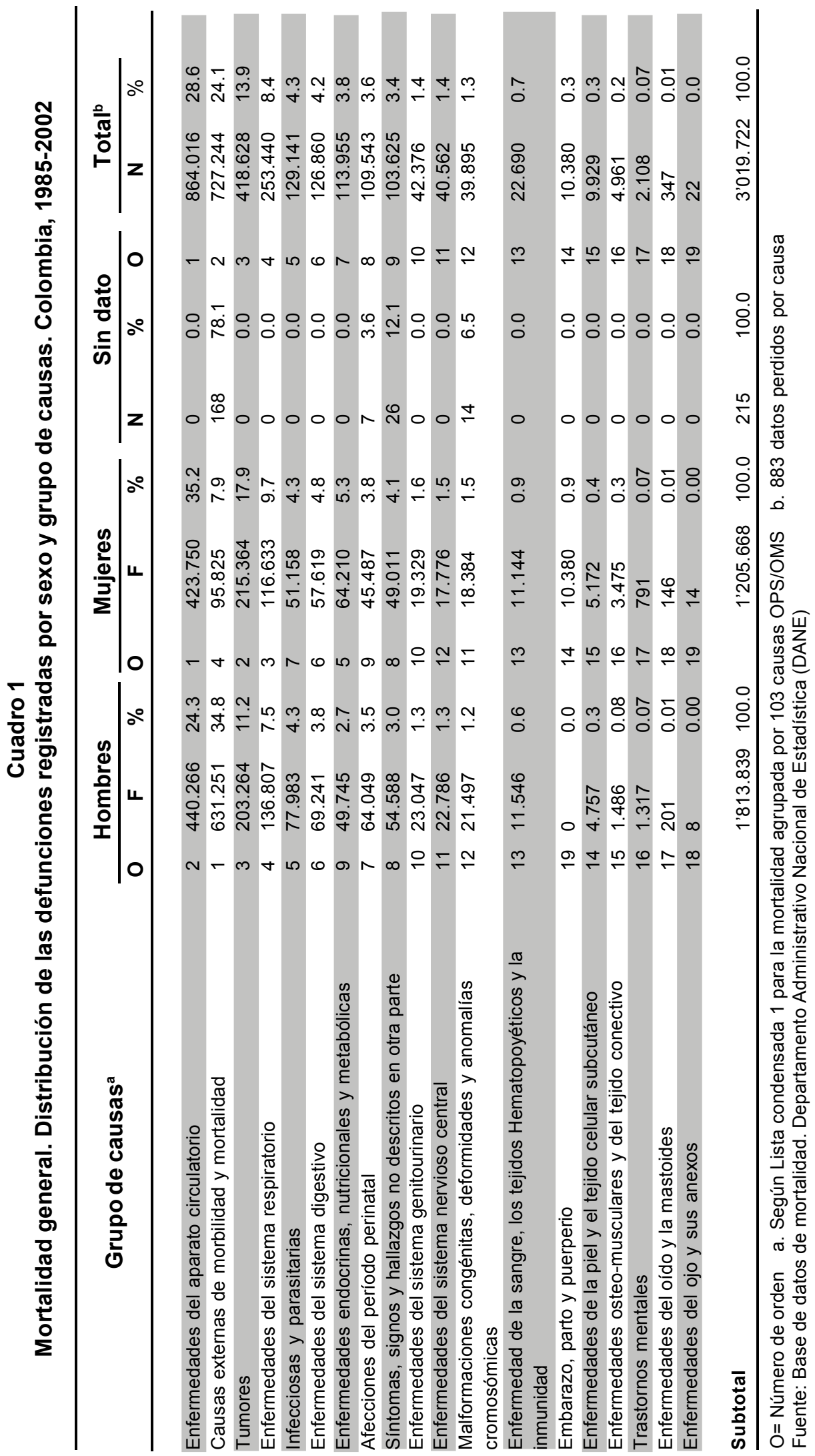

las entidades cardiovasculares, los tumores y las complicaciones respiratorias. El Cuadro 2 muestra la distribución anual de las tasas ajustadas por cien mil personas y la Gráfica 1 la RCM por grupo de causas; los datos sugieren que la mortalidad general (MG) disminuyó durante el período de forma lenta e irregular. La tendencia de la RCM (Gráfica 1) muestra que el riesgo de morir disminuyó en la población general durante todo el período; entre los hombres, el riesgo de morir se redujo en $19 \%$, aunque presentó incrementos significativos en 1991 y 1999 , que coinciden con picos en la mortalidad por homicidios; entre las mujeres la disminución del riesgo fue superior $(43 \%)$ y más pronunciada que entre los hombres.

Una elevada proporción de las defunciones registradas durante el período se ajustó a los criterios de ME (Cuadro 3); la ME representó $75.3 \%$ (95\% CI: 75.2-75.4) de todas las defunciones; al analizar la estructura interna de la ME en la población general, predominaron las muertes evitables por atención médica (49.6\%; 95\% CI: 49.6-49.7) y por medidas mixtas (47.9\%; 95\% CI: 47.948.0); las muertes evitables por prevención primaria o por saneamiento ambiental fueron relativamente raras y se presentaron en una proporción menor de $2.5 \%$.

La estructura de la ME tuvo diferencias por sexos (Cuadro 3). Las muertes evi- 
Cuadro 2

Distribución de la mortalidad según evitabilidad y sexo.

Tasas ajustadas (TA) por cien mil. Colombia.1985-2002

\begin{tabular}{|c|c|c|c|c|c|c|c|c|c|c|c|c|c|c|c|}
\hline \multirow{3}{*}{ Año } & \multicolumn{15}{|c|}{ Causas de mortalidad } \\
\hline & \multicolumn{3}{|c|}{ General } & \multicolumn{3}{|c|}{ Evitables } & \multicolumn{3}{|c|}{ No evitables } & \multicolumn{3}{|c|}{ Mal definidas } & \multicolumn{3}{|c|}{ Otras } \\
\hline & $\mathbf{H}$ & $\mathbf{M}$ & Ambos & $\overline{\mathrm{H}}$ & $\mathbf{M}$ & Ambos & $\overline{\mathbf{H}}$ & $\mathbf{M}$ & Ambos & $\mathrm{H}$ & $\mathbf{M}$ & Ambos & $\overline{\mathrm{H}}$ & $\mathbf{M}$ & Ambos \\
\hline 1985 & 824 & 558 & 683 & 607 & 383 & 489 & 114 & 93 & 103 & 44 & 35 & 39 & 60 & 47 & 53 \\
\hline 1986 & 765 & 503 & 627 & 564 & 338 & 445 & 108 & 89 & 97 & 38 & 31 & 34 & 56 & 45 & 50 \\
\hline 1987 & 777 & 505 & 633 & 575 & 341 & 452 & 108 & 89 & 97 & 37 & 29 & 33 & 58 & 46 & 51 \\
\hline 1988 & 769 & 488 & 621 & 579 & 335 & 450 & 104 & 87 & 95 & 32 & 25 & 29 & 54 & 42 & 48 \\
\hline 1989 & 757 & 475 & 608 & 575 & 325 & 444 & 104 & 87 & 95 & 28 & 23 & 25 & 51 & 40 & 45 \\
\hline 1990 & 741 & 457 & 591 & 562 & 316 & 433 & 101 & 82 & 91 & 28 & 20 & 24 & 50 & 39 & 44 \\
\hline 1991 & 767 & 454 & 602 & 587 & 312 & 443 & 104 & 84 & 93 & 28 & 21 & 24 & 48 & 37 & 42 \\
\hline 1992 & 768 & 452 & 601 & 594 & 316 & 448 & 99 & 79 & 88 & 27 & 20 & 23 & 49 & 37 & 43 \\
\hline 1993 & 753 & 446 & 591 & 585 & 313 & 442 & 95 & 77 & 85 & 28 & 20 & 23 & 46 & 36 & 41 \\
\hline 1994 & 732 & 436 & 575 & 568 & 307 & 431 & 91 & 74 & 82 & 26 & 19 & 22 & 46 & 35 & 40 \\
\hline 1995 & 715 & 434 & 566 & 559 & 310 & 428 & 90 & 74 & 81 & 25 & 17 & 21 & 41 & 32 & 36 \\
\hline 1996 & 715 & 427 & 563 & 566 & 309 & 430 & 88 & 73 & 80 & 22 & 16 & 19 & 38 & 30 & 34 \\
\hline 1997 & 682 & 410 & 538 & 520 & 286 & 397 & 84 & 66 & 74 & 28 & 19 & 23 & 51 & 39 & 45 \\
\hline 1998 & 678 & 408 & 535 & 525 & 288 & 400 & 79 & 64 & 71 & 19 & 13 & 16 & 55 & 43 & 49 \\
\hline 1999 & 695 & 416 & 547 & 543 & 299 & 414 & 81 & 63 & 71 & 13 & 9 & 11 & 58 & 44 & 51 \\
\hline 2000 & 691 & 406 & 540 & 540 & 288 & 407 & 80 & 64 & 71 & 12 & 8 & 10 & 60 & 46 & 52 \\
\hline 2001 & 662 & 390 & 518 & 515 & 275 & 388 & 76 & 61 & 68 & 11 & 7 & 9 & 60 & 47 & 53 \\
\hline 2002 & 670 & 394 & 524 & 518 & 272 & 388 & 80 & 64 & 72 & 11 & 7 & 9 & 62 & 51 & 56 \\
\hline
\end{tabular}

$\mathrm{H}=$ Hombre $\quad \mathrm{M}=$ Mujer

Cuadro 3

Evitabilidad de la mortalidad. Distribución porcentual por sexo y grupo de causas. Colombia. 1985-2002

\begin{tabular}{lccc}
\hline Grupo de causas & $\begin{array}{c}\text { Ambos sexos } \\
\mathbf{N}=\mathbf{3} \mathbf{0 2 0 . 3 7 0 ( 1 0 0 \% )}\end{array}$ & $\begin{array}{c}\text { Hombres } \\
\mathbf{N = 1} \mathbf{8 1 4 . 4 5 5 ( 6 2 . 8 \% )}\end{array}$ & $\begin{array}{c}\text { Mujeres } \\
\mathbf{N = 1} \mathbf{2 0 5 . 9 1 5 ( 3 7 . 2 \% )}\end{array}$ \\
\hline Mortalidad evitable & 75.3 & 78.8 & 70.1 \\
\hline Por prevención & 0.3 & 0.2 & 0.3 \\
Por atención médica & 37.4 & 30.8 & 47.3 \\
Por atención ambiental & 1.6 & 1.4 & 1.8 \\
Por medidas mixtas & 36.0 & 46.4 & 20.7 \\
Mortalidad no evitable & 13.1 & 11.0 & 16.1 \\
Causas mal definidas & 3.5 & 3.1 & 4.2 \\
Otras causas no clasificables & 8.1 & 7.1 & 9.7 \\
Total & 100.0 & 100.0 & 100.0 \\
\hline
\end{tabular}




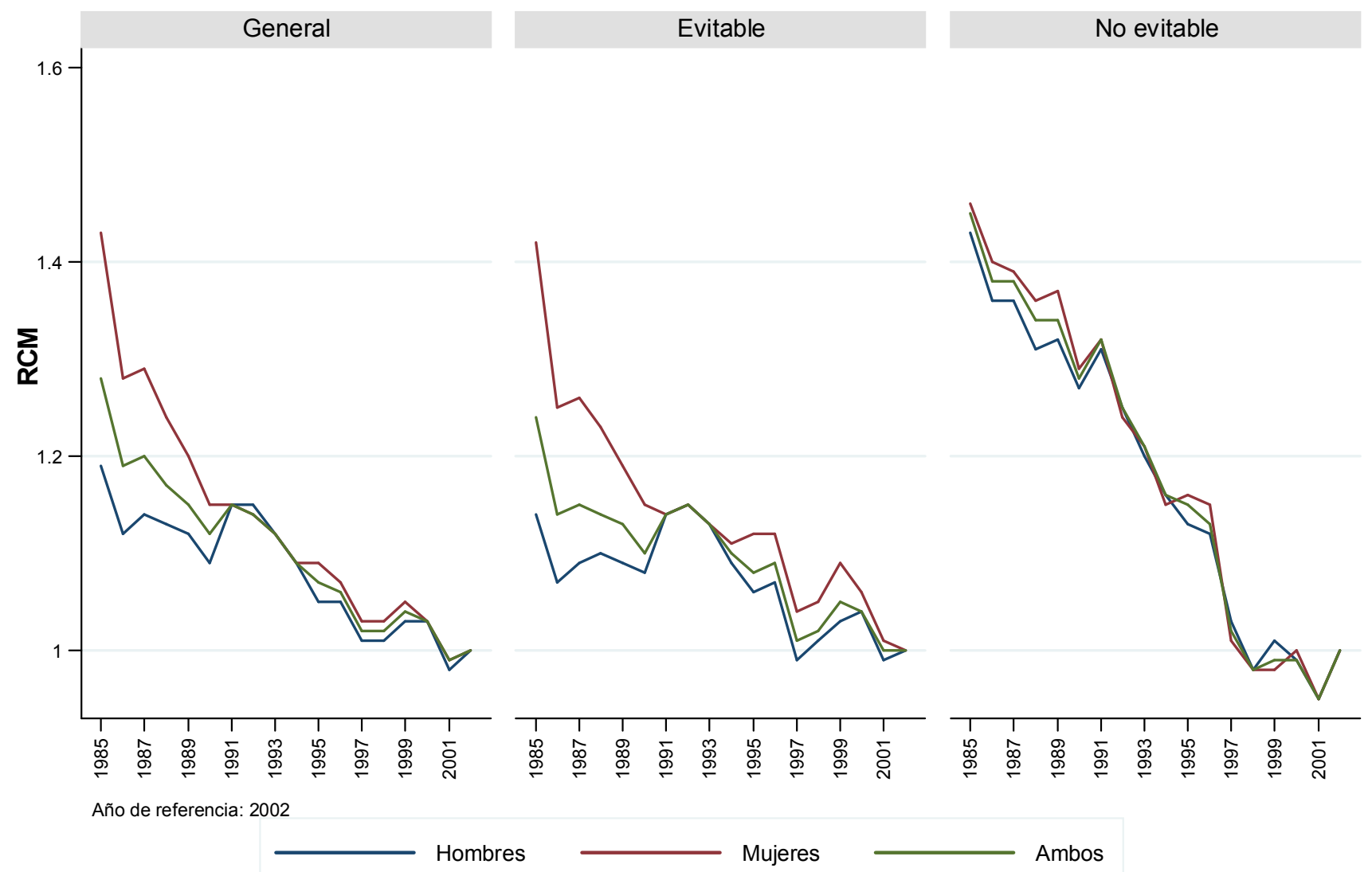

Gráfica 1. Razón comparativa de mortalidad RCM por sexo y año. Colombia. 1985-2002

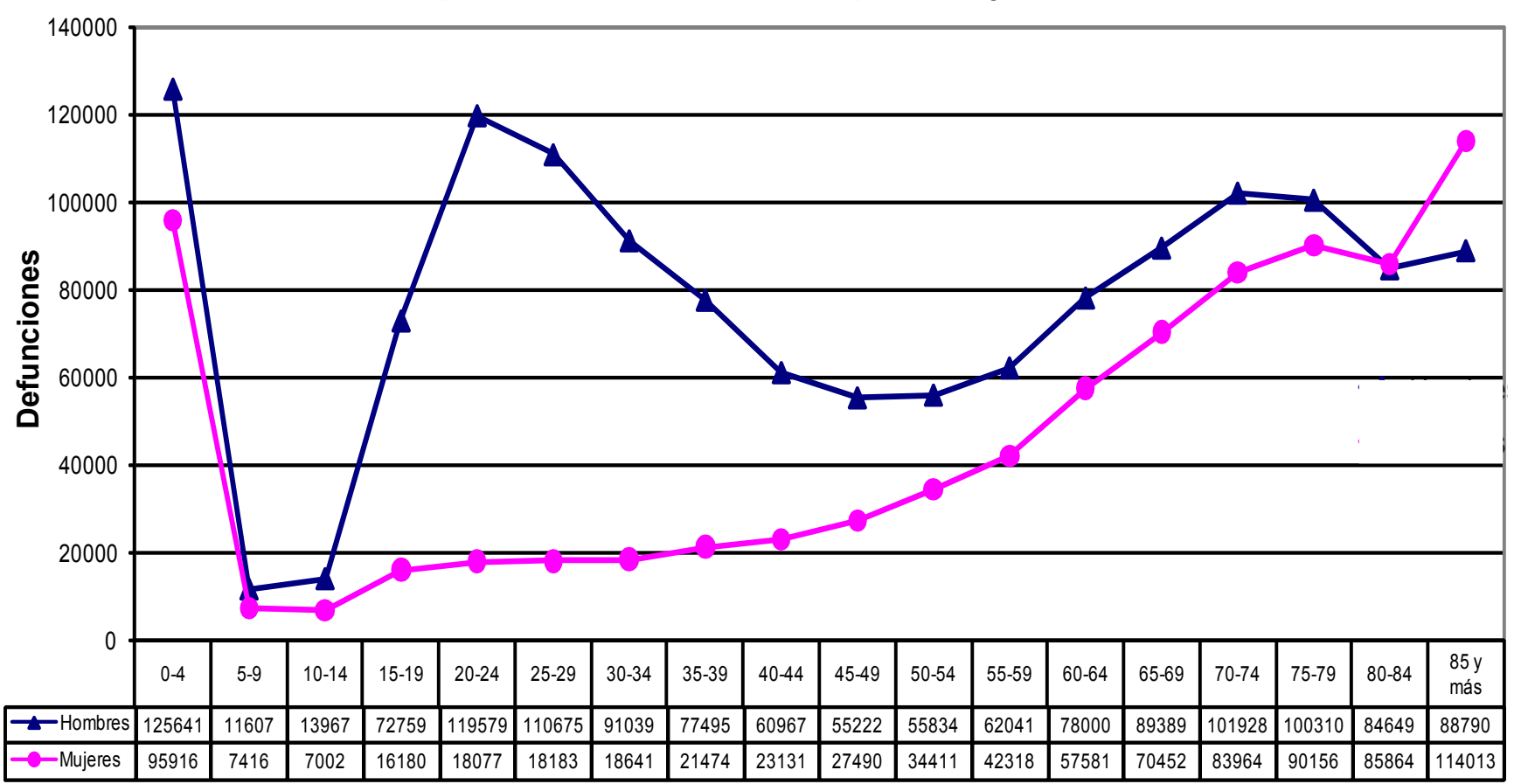

Grupo quinquenal de edad

Gráfica 2. Defunciones evitables por sexo y grupo de edad. Colombia. 1985-2002 


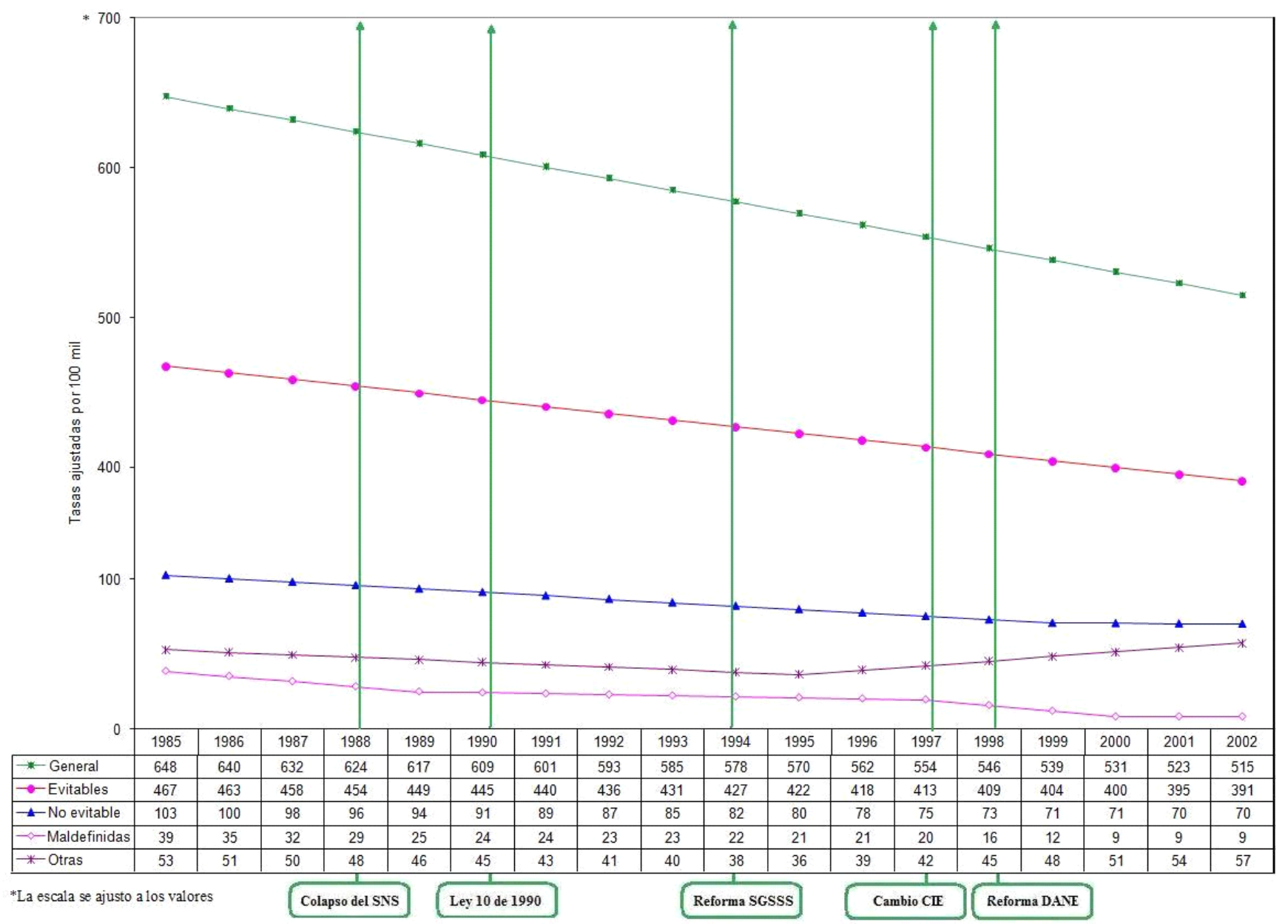

Tasas ajustadas contra la población estándar de la OMS. SNS= Sistema Nacional de Salud SGSSS= Sistema General de Seguridad Social en Salud DANE = Departamento Administrativo Nacional de Estadísticas $\mathrm{CIE}=$ Clasificación Internacional de Enfermedades

\section{Gráfica 3. Tendencia anual de la mortalidad según evitabilidad. Tasas ajustadas por 100 mil. Análisis de joinpoint. Colombia. 1985-2002}

tables $(62.8 \%)$ ocurrieron en su mayoría entre los varones. En este grupo la ME representó $78.8 \%$ (95\% CI: 78.7-78.8) de las defunciones, especialmente en el subgrupo de 15 a 54 años que aportaron $28.3 \%$ de todas las defunciones evitables del país. Entre las mujeres la proporción de ME fue 70.1\% (95\% CI: 70.0-70.1). En los hombres la proporción de ME por medidas mixtas, categoría que incluye los homicidios y accidentes, predomina sobre la ME por atención médica. Entre las mujeres, la ME ocurre sobre todo por atención médica, a expensas de causas cardiovasculares. La distribución de la ME registrada en el período varió también con la edad (Gráfica 2). Entre los hombres las defunciones evitables hicieron una curva con tres picos: fueron más comunes en los menores de cinco años; disminuyeron bruscamente durante la edad escolar y ascendieron de nuevo durante la adolescencia hasta un pico máximo entre los 20-24 años a expensas de las causas externas; desde la tercera década disminuyeron de nuevo hasta los 50-54 años, cuando comenzaron un tercer ascenso que hizo su pico alrededor de los 70 años, a expensas sobre todo de las causas cardiovasculares. Entre las mujeres la ME presenta una frecuencia alta en el primer quinquenio; disminuye bruscamente hacia la edad escolar y desde entonces aumenta de modo progresivo con la edad, sin que se observe el pico de defunciones evitables que afecta a los hombres adultos jóvenes y que obedece a las causas externas. 
El comportamiento anual de la mortalidad la clasificación de evitabilidad se muestra en la Gráfica 3. El Cuadro 4 muestra los análisis de joinpoint de las tasas ajustadas por sexo y grupo de causas; para cada serie de datos se presentan los momentos de inflexión y la caracterización de cada período e indica los años de inicio y terminación, las tasas inicial y final y la tasa de variación con respecto a la tasa basal del segmento analizado.

Al considerar la población en su conjunto se observa que las tasas ajustadas de MG por todas las causas (Cuadro 4) disminuyen entre 1985 y 2002 con una cifra anual de disminución de $1.1 \%$ con respecto a la tasa inicial; la reducción de la MG no muestra puntos de inflexión significativos durante el período. Un patrón similar se observó en el subgrupo de los hombres donde la $\mathrm{MG}$ se redujo a un ritmo promedio anual de 7.9 defunciones por 100 mil varones sin puntos de inflexión significativos. En el subgrupo de las mujeres, también se observó una tendencia a la disminución a lo largo de todo el período, pero con dos patrones diferentes: desde el inicio de la serie y hasta 1989 , el riesgo de morir entre las mujeres se redujo 2.4 veces más rápido que entre los hombres; desde 1990 la tendencia cambia y las mujeres muestran una desaceleración significativa en el riesgo de morir que se hace 3.1 veces más lenta que en el período anterior y más lenta que entre los hombres. $\mathrm{Al}$ analizar la tendencia de la ME con base en la RCM (Gráfica 1) se evidencia una disminución irregular de los riesgos anuales de morir por causas evitables, tanto en el grupo de los hombres como entre las mujeres; a pesar de la tendencia al descenso, entre los varones se observan dos picos en el riesgo: el primero entre 1991 y 1993, especialmente a expensas de las causas externas, que coincide con la guerra por el narcotráfico, y el segundo entre 1999 y 2000 acompañado de un aumento en los homicidios y en las complicaciones cardiovasculares. Entre las mujeres el riesgo de morir por causas evitables presentó también un pico entre 1998 y 2000, acompañado de un aumento en las muertes evitables por atención médica.

Los análisis de joinpoint para las tasas ajustadas de ME (Cuadro 4) confirman que este grupo de defunciones ha disminuido en Colombia de manera regular desde el comienzo de la serie con una proporción anual de cambio de $-0.9 \%$ frente a la tasa de 1985 ; la tasa de reducción es mayor y más rápida que la observada en la categoría de causas no evitables. El análisis de joinpoint no detecta cambios significativos en la tendencia de la ME cuando se considera globalmente, pero en cambio sí muestra variaciones importantes por sexo y grupo de causas (Cuadro 4). En la población general y entre los varones, la ME disminuye de forma similar a la MG por todas las causas, sin cambios significativos durante el período observado. No ocurre lo mismo entre las mujeres donde el descenso en la $\mathrm{ME}$ es continuo y sin cambios significativos, mientras la disminución en la mortalidad por todas las causas se desacelera a partir de 1990, especialmente a expensas del grupo de otras enfermedades cardiovasculares y tumores no evitables.

\section{DISCUSIÓN}

Con el fin de descubrir variaciones en la cobertura o la calidad de los datos que se podrían atribuir al cambio en los sistemas de clasificación (1997) o registro (1998), se analizaron las tendencias de la MG y de la proporción de MCM. Durante el período estudiado no se observaron inflexiones significativas en la tendencia de la $\mathrm{MG}$, localizadas en la proximidad de 1998, que pudieran reflejar cambios en la cobertura del registro atribuibles a los ajustes implantados en este año por el DANE; por el contrario, las tasas continuaron su descenso sin variaciones, tanto en hombres como en mujeres. Las tasas ajustadas y el peso proporcional de la MCM reflejan un mejoramiento progresivo y relativamente sostenido en la clasificación de los registros desde el comienzo de la serie hasta 1996; al año siguiente, y en coincidencia con la implantación de la CIE-10, la proporción de MCM ascendió bruscamente; este incremento pudo corresponder a la inexperiencia de los sistemas de registro y clasificación con los nuevos criterios de la CIE-10, y se acompañó de un descenso súbito en el peso proporcional de la $\mathrm{ME}$, categoría que pudo presentar un subregistro temporal a expensas de la mala clasificación. El análisis de joinpoint (Cuadro 4) confirmó que la tasa por MCM disminuía en Colombia desde el comienzo, tanto en hombres como en mujeres, y reveló además una desaceleración significativa de las tasas de MCM entre 1990 y 1997 que no se percibía en los otros análisis; el comienzo de estos cambios coincide con la agudización de la crisis financiera del Sistema Nacional de Salud (1988) y con su desmonte a partir de la Ley 10 de 1990 que descentralizó el sector. En este 


\section{Cuadro 4}

Tendencia de la mortalidad según evitabilidad por sexo. Análisis de Joinpoint para tasas ajustadas. Colombia. 1985-2002

\begin{tabular}{|c|c|c|c|c|c|c|c|c|c|c|c|c|}
\hline \multirow[t]{2}{*}{ Grupo de causas } & \multirow[t]{2}{*}{ Período } & \multicolumn{2}{|c|}{ Tasa } & \multirow[t]{2}{*}{ b } & \multirow[t]{2}{*}{ Período } & \multicolumn{2}{|c|}{ Tasa } & \multirow[t]{2}{*}{ b } & \multirow[t]{2}{*}{ Período } & \multicolumn{2}{|c|}{ Tasa } & \multirow[t]{2}{*}{ b } \\
\hline & & inicial & final & & & inicial & final & & & inicial & final & \\
\hline Mortalidad general & $1985-02$ & 647.71 & 515.14 & $-7.80^{*}$ & & & & & & & & \\
\hline En varones & 1985-02 & 798.77 & 664.12 & $-7.92 *$ & & & & & & & & \\
\hline En mujeres & $1985-89$ & 541.34 & 467.24 & $-18.52 *$ & 1990-02 & 461.30 & 390.08 & $-5.93 *$ & & & & \\
\hline Mortalidad no evitable & $1985-99$ & 102.77 & 70.91 & $-2.28 *$ & $2000-02$ & 70.69 & 70.26 & -0.21 & & & & \\
\hline En varones & 1985-02 & 111.94 & 75.24 & $-2.16^{*}$ & & & & & & & & \\
\hline En mujeres & 1985-02 & 92.43 & 59.88 & $-1.91^{*}$ & & & & & & & & \\
\hline Causas mal definidas & $1985-89$ & 38.77 & 25.13 & $-3.41 *$ & $1990-97$ & 24.47 & 19.86 & $-0.66^{*}$ & 1998-00 & 16.10 & 8.57 & $-3.77 *$ \\
\hline En varones & $1985-89$ & 43.39 & 28.77 & $-3.65^{*}$ & $1990-97$ & 28.19 & 24.13 & $-0.58 *$ & 1998-00 & 19.63 & 10.61 & -4.51 \\
\hline En mujeres & $1985-89$ & 34.99 & 22.00 & $-3.25^{*}$ & $1990-97$ & 21.30 & 16.41 & $-0.70^{*}$ & 1998-00 & 13.22 & 8.63 & $-3.19 *$ \\
\hline \multicolumn{13}{|l|}{ Otras causas no clasificadas } \\
\hline En varones & $1985-95$ & 52.77 & 36.49 & $-1.63^{*}$ & 1996-02 & 39.48 & 57.38 & $2.98 *$ & & & & \\
\hline En mujeres & $1985-96$ & 59.08 & 40.78 & $-1.66^{*}$ & 1997-99 & 47.19 & 60.02 & $6.41 *$ & $2000-02$ & 60.41 & 61.20 & 0.39 \\
\hline Mortalidad evitable & $1985-95$ & 46.98 & 32.01 & $-1.50 *$ & 1996-02 & 34.74 & 51.11 & $2.73 *$ & & & & \\
\hline En varones & 1985-02 & 467.39 & 390.85 & $-4.50 *$ & & & & & & & & \\
\hline En mujeres & 1985-02 & 597.09 & 522.49 & $-4.38 *$ & & & & & & & & \\
\hline $\begin{array}{l}\text { Muertes evitables por } \\
\text { prevención }\end{array}$ & 1985-02 & 349.53 & 273.38 & $-4.47 *$ & & & & & & & & \\
\hline En varones & $1985-95$ & 2.77 & 0.78 & $-0.19^{*}$ & 1996-02 & 0.79 & 0.81 & -0.00 & & & & \\
\hline En mujeres & $1985-96$ & 2.83 & 0.90 & $-0.17 *$ & 1997-02 & 0.91 & 0.92 & -0.00 & & & & \\
\hline Tétanos & $1985-95$ & 2.61 & 0.65 & $-0.19^{*}$ & 1996-02 & 0.65 & 0.70 & -0.00 & & & & \\
\hline En varones & $1985-93$ & 0.54 & 0.14 & $-0.05 *$ & 1994-02 & 0.13 & 0.09 & -0.00 & & & & \\
\hline En mujeres & $1985-93$ & 0.58 & 0.18 & $-0.05^{*}$ & 1994-02 & 0.18 & 0.13 & -0.01 & & & & \\
\hline Tos ferina & $1985-93$ & 0.49 & 0.09 & $-0.05^{*}$ & 1994-02 & 0.08 & 0.06 & -0.00 & & & & \\
\hline En varones & $1985-92$ & 0.34 & 0.05 & $-0.04 *$ & 1993-02 & 0.05 & 0.05 & 0.00 & & & & \\
\hline En mujeres & $1985-92$ & 0.33 & 0.03 & $-0.04 *$ & 1993-02 & 0.03 & 0.05 & 0.00 & & & & \\
\hline \multicolumn{12}{|l|}{ Infecciones de transmisión } & \\
\hline En varones & 1985-02 & 0.17 & 0.08 & $-0.01 *$ & & & & & & & & \\
\hline En mujeres & 1985-02 & 0.18 & 0.10 & $-0.01 *$ & & & & & & & & \\
\hline $\begin{array}{l}\text { Muertes Evitables por atención } \\
\text { médica }\end{array}$ & 1985-02 & 0.15 & 0.06 & $-0.01 *$ & & & & & & & & \\
\hline En varones & 1985-02 & 265.67 & 224.61 & $-2.42 *$ & & & & & & & & \\
\hline En mujeres & 1985-02 & 292.98 & 257.18 & $-2.11 *$ & & & & & & & & \\
\hline Muertes cardiovasculares en & 1985-02 & 244.06 & 199.76 & $-2.60 *$ & & & & & & & & \\
\hline mayores de 20 años & 1985-02 & 156.01 & 127.21 & $-1.69 *$ & & & & & & & & \\
\hline En varones & 1985-02 & 178.82 & 150.23 & $-1.68 *$ & & & & & & & & \\
\hline En mujeres & 1985-02 & 136.86 & 108.57 & $-1.66^{*}$ & & & & & & & & \\
\hline Cáncer de cuello del útero & $1985-91$ & 10.39 & 8.54 & $-0.39^{*}$ & $1992-96$ & 8.83 & 9.99 & 0.29 & 1997-02 & 9.78 & 8.75 & $-0.21^{*}$ \\
\hline Cáncer de mama en mujeres & $1985-02$ & 7.28 & 9.19 & $0.11^{*}$ & & & & & & & & \\
\hline Cáncer de próstata & $1985-02$ & 8.70 & 14.81 & $0.36^{*}$ & & & & & & & & \\
\hline Muertes evitables por & & & & & & & & & & & & \\
\hline saneamiento & $1985-89$ & 15.39 & 8.61 & $-1.69 *$ & 1990-02 & 8.26 & 4.10 & $-0.35 *$ & & & & \\
\hline En varones & $1985-89$ & 16.71 & 9.54 & $-1.79 *$ & 1990-02 & 9.16 & 4.54 & $-0.38 *$ & & & & \\
\hline En mujeres & $1985-89$ & 14.05 & 7.73 & $-1.58 *$ & 1990-02 & 7.41 & 3.65 & $-0.31 *$ & & & & \\
\hline Diarreas en menores de 5 años & $1985-94$ & 9.12 & 3.23 & $-0.65 *$ & 1995-02 & 3.13 & 2.39 & -0.11 & & & & \\
\hline En varones & $1985-93$ & 10.0 & 3.93 & $-0.76^{*}$ & 1994-02 & 3.77 & 2.47 & $-0.16^{*}$ & & & & \\
\hline En mujeres & $1985-94$ & 8.39 & 2.86 & $-0.61 *$ & 1995-02 & 2.78 & 2.20 & -0.08 & & & & \\
\hline Muertes evitables por medidas & 1985-02 & 186.48 & 162.24 & $-1.43 *$ & & & & & & & & \\
\hline mixtas & $1985-92$ & 265.89 & 299.13 & $4.75^{*}$ & 1993-97 & 290.73 & 257.10 & $-8.41^{*}$ & 1998-02 & 258.14 & 262.30 & 1.04 \\
\hline En varones & 1985-02 & 92.11 & 70.19 & $-1.29^{*}$ & & & & & & & & \\
\hline En mujeres & $1985-92$ & 39.31 & 80.33 & $5.86^{*}$ & 1993-97 & 75.01 & 53.77 & $-5.31 *$ & 1998-02 & 56.75 & 68.67 & $2.98 *$ \\
\hline Homicidios & $1985-92$ & 75.24 & 151.75 & $10.93 *$ & 1993-97 & 141.76 & 101.81 & $-9.99 *$ & 1998-02 & 107.22 & 128.85 & $5.41 *$ \\
\hline En varones & 1985-92 & 5.01 & 11.86 & $0.98^{*}$ & 1993-97 & 11.07 & 7.89 & $-0.79^{*}$ & 1998-02 & 8.46 & 10.73 & $0.57 *$ \\
\hline En mujeres & $1985-94$ & 0.02 & 0.05 & 0.00 & 1995-02 & 0.09 & 0.38 & $0.04 *$ & & & & \\
\hline Dengue & $1985-92$ & 0.04 & 0.00 & -0.00 & 1993-02 & 0.05 & 0.44 & $0.04 *$ & & & & \\
\hline En varones & 1985-02 & -0.04 & 0.18 & $0.01 *$ & & & & & & & & \\
\hline En mujeres & & & & & & & & & & & & \\
\hline Malaria & $1985-95$ & 1.10 & 0.22 & $-0.09 *$ & 1996-02 & 0.25 & 0.41 & 0.03 & & & & \\
\hline En varones & 1985-94 & 1.42 & 0.31 & $-0.12 *$ & 1995-02 & 0.33 & 0.46 & 0.02 & & & & \\
\hline En mujeres & $1985-94$ & 0.87 & 0.22 & $-0.07 *$ & 1995-02 & 0.22 & 0.26 & 0.01 & & & & \\
\hline Desnutrición & $1985-94$ & 3.75 & 2.51 & $-0.14 *$ & $1995-99$ & 3.11 & 5.48 & $0.59 *$ & 1999-02 & 5.23 & 4.71 & -0.26 \\
\hline En varones & $1985-94$ & 4.14 & 2.78 & -0.15 & 1995-99 & 3.48 & 6.27 & $0.70^{*}$ & 1999-02 & 5.90 & 5.16 & -0.37 \\
\hline En mujeres & 1985-94 & 3.40 & 2.26 & $-0.13^{*}$ & 1995-99 & 2.77 & 4.81 & $0.51 *$ & 1999-02 & 4.67 & 4.38 & -0.14 \\
\hline
\end{tabular}




\section{Cuadro 4}

Tendencia de la mortalidad según evitabilidad por sexo. Análisis de joinpoint para tasas ajustadas. Colombia. 1985-2002 (continuación)

\begin{tabular}{|c|c|c|c|c|c|c|c|c|c|c|c|c|}
\hline \multirow[t]{2}{*}{ Grupo de causas } & \multirow[t]{2}{*}{ Período } & \multicolumn{2}{|c|}{ Tasa } & \multirow[t]{2}{*}{ b } & \multirow[t]{2}{*}{ Período } & \multicolumn{2}{|c|}{ Tasa } & \multirow[t]{2}{*}{ b } & \multirow[t]{2}{*}{ Período } & \multicolumn{2}{|c|}{ Tasa } & \multirow[t]{2}{*}{ b } \\
\hline & & inicial & final & & & inicial & final & & & inicial & final & \\
\hline \multicolumn{13}{|c|}{ Desnutrición en menores } \\
\hline En varones & $1985-94$ & 1.87 & 0.56 & $-0.15^{*}$ & 1995-02 & 0.75 & 2.04 & $0.18^{*}$ & & & & \\
\hline En mujeres & $1985-94$ & 1.68 & 0.52 & $-0.13^{*}$ & 1995-02 & 0.69 & 1.90 & $0.17^{*}$ & & & & \\
\hline \multicolumn{13}{|c|}{ Respiratorias en menores } \\
\hline En varones & $1985-93$ & 8.40 & 4.64 & $-0.47 *$ & 1994-02 & 4.53 & 3.65 & $-0.11^{*}$ & & & & \\
\hline En mujeres & $1985-93$ & 7.54 & 4.03 & $-0.44 *$ & 1994-02 & 3.94 & 3.20 & $-0.09 *$ & & & & \\
\hline Tuberculosis & $1985-91$ & 8.59 & 4.68 & $-0.65^{*}$ & 1992-02 & 4.57 & 3.45 & $-0.11 *$ & & & & \\
\hline En varones & $1985-90$ & 12.31 & 7.06 & $-1.05 *$ & 1991-01 & 6.89 & 5.09 & $-0.16^{*}$ & & & & \\
\hline En mujeres & $1985-91$ & 5.72 & 2.94 & $-0.46^{*}$ & 1992-01 & 2.86 & 2.09 & $-0.08 *$ & & & & \\
\hline Obstétricas & $1985-96$ & 3.73 & 1.78 & $-0.18^{*}$ & 1997-00 & 2.15 & 3.24 & $0.36^{*}$ & 2001-02 & 2.73 & 2.23 & -0.50 \\
\hline Perinatales & $1985-97$ & 17.85 & 11.81 & $-0.50 *$ & 1998-00 & 13.74 & 17.60 & $1.93^{*}$ & 2001-02 & 15.02 & 12.44 & $-2.58 *$ \\
\hline En varones & $1985-97$ & 20.33 & 13.82 & $-0.54 *$ & 1998-00 & 15.85 & 19.90 & 2.03 & 2001-02 & 17.04 & 14.17 & $-2.86^{*}$ \\
\hline En mujeres & $1985-97$ & 15.23 & 9.78 & $-0.45^{*}$ & $1998-00$ & 11.60 & 15.24 & $1.82 *$ & 2001-02 & 12.91 & 10.58 & $-2.33^{*}$ \\
\hline
\end{tabular}

Tasas por cien mil b: variación anual de la tasa en muertes por 100 mil. * Variación significativamente diferente de cero ( $p<0.05)$

contexto y en presencia de una $\mathrm{MG}$ con tendencia sostenida a la disminución, la desaceleración observada en las tasas de MCM durante este período pudiera reflejar un deterioro de la red de servicios para clasificar las defunciones, o del sistema de estadísticas vitales o de ambos, cuyo principio pudo darse alrededor de 1990; los diversos análisis apuntan a que hubo un deterioro en el sistema de clasificación de la mortalidad entre 1990 y 1997 y quizá un subregistro concomitante de la ME; sin embargo, la comprobación de las causas que dieron lugar a este fenómeno escapa a los alcances del presente estudio. Los distintos análisis de la MG no sugieren reducciones significativas durante el período que reflejen un mejoramiento atribuible a la aplicación de la Ley 10 de 1990 o de la Ley 100 de 1993. Por el contrario, entre las mujeres, la desaceleración de la tasa de mortalidad observada desde 1990, sugiere un deterioro en el control de las causas de muerte que actúan sobre este grupo y confluyen en dicho período.

El análisis de la ME debe realizarse en el contexto epidemiológico de Colombia y tener en cuenta las propiedades de los instrumentos utilizados para medirla. La proporción de ME que se encontró en este estudio es mucho mayor que la ME observada en países que utilizan los criterios de Holland y ello obedece a que esta última clasificación se enfoca en causas evitables por atención médica ${ }^{8}$. La proporción de ME obtenida a partir del ICME excede también a la encontrada en otras regiones de América Latina durante el mismo período; sin embargo, la estructura por causas es similar. En algunos estados de México la ME, establecida con los criterios de Taucher, representaba para la década de 1980 , entre $50 \%$ y $60 \%$ de las defunciones, a expensas de accidentes y violencia y las muertes evitables por diagnóstico y tratamiento; las muertes evitables por saneamiento y por prevención fueron menos frecuentes ${ }^{18}$. Algo similar se observó en Guatemala donde, con los criterios de Taucher, la ME representó más de $50 \%$ de las defunciones registradas, especialmente por medidas mixtas y medidas de saneamiento ambiental.En Medellín (Colombia) las defunciones evitables registradas pasaron de $54.5 \%$ en 1982 a $73 \%$ en 1992 y obedecieron en su mayoría a accidentes y homicidios. La mayor proporción de MEobservada en este estudio puede explicarse en razón de que el ICME incluyó causas de muerte importantes en el perfil epidemiológico de Colombia que no están incluidas en el listado de Taucher (en Gómez-Arias ${ }^{8}$ ) y cuya dinámica se refleja en los análisis por grupos de causas (Cuadro 4).

En el país, la ME presentó diferencias por sexo, explicables en gran parte por el peso que tienen las causas externas en la estructura de la mortalidad masculina. Considerada en su conjunto, la ME ha disminuido lentamente en Colombia desde el principio de la serie con una cifra $\mathrm{AC}$ de $-0.9 \%$, y su tendencia global no presenta inflexiones que pudieran reflejar el efecto favorable, a corto plazo, de las reformas sanitarias. Sin 
embargo, el análisis de cada grupo de causas por separado mostró un comportamiento heterogéneo a lo largo del período, y sugiere que los determinantes que conducen a la muerte experimentan dinámicas propias y no responden de la misma manera a las políticas sanitarias. Con base en los análisis de joinpoint (Cuadro4) el estudio identificó siete patrones de inflexión que pudieran reflejar, de manera diferente, el efecto de las políticas sanitarias, vigentes durante el período:

- Patrón 1. Causas que disminuyeron sin variación significativa desde el comienzo de la serie, en cuya tendencia no se reflejan cambios atribuibles a corto plazo a las reformas sanitarias de 1990 y 1993. Este es el patrón que muestran las defunciones cuando se analizan por grandes grupos: mortalidad general en los hombres, ME en hombres y en mujeres y mortalidad no evitable en hombres y en mujeres. Este mismo patrón se repite en algunos subgrupos de causas: tosferina en mujeres, infecciones de transmisión sexual (ITS) exceptuando VIH en hombres y en mujeres, ME por atención médica en hombres y en mujeres, mortalidad por cardiovasculares en mayores de 20 años tanto en hombres como en mujeres, y muertes evitables por medidas mixtas en mujeres. A este patrón se ajustan casi todas las defunciones registradas en el país y no se encontró evidencia de que las políticas sociales o las reformas sanitarias aplicadas en Colombia durante el período hayan modificado significativamente su descenso progresivo, a pesar del aumento en la inversión social en salud de los últimos años.

- Patrón 2. Causas que aumentaron sin variación durante todo el período y donde tampoco se observan cambios que reflejen los efectos de las políticas sociales y sanitarias. Este es el caso de las muertes por cáncer de próstata, y las muertes por cáncer de mama y por dengue entre las mujeres. El análisis sugiere que ni el modelo del Sistema Nacional de Salud ni el modelo de competencia regulada generaron, durante el período, inflexiones significativas en los riesgos de morir por estas causas, las cuales aumentaron en el país a un ritmo sostenido desde el principio de la serie.

- Patrón 3. Causas que venían en descenso y cuya disminución se ha desacelerado significativamente desde comienzos de 1990 y sugieren un deterioro en las medidas que eran efectivas hasta el momento del cambio. En esta categoría, los primeros indicadores en deteriorarse fueron la mortalidad general en mujeres
(1990), una inflexión que no se había encontrado en estudios previos ${ }^{8}$, y las evitables por saneamiento en hombres y mujeres (1990); luego se desaceleran la mortalidad por tuberculosis en hombres (1991) y en mujeres (1992), el tétanos en mujeres y en hombres (1994), las muertes por diarrea en menores de 5 años entre los niños (1994) y entre las niñas (1995), y las muertes por infección respiratoria aguda en niños y en niñas (1994). Estos cambios anteceden a las reformas implantadas en 1995 por la reglamentación de la Ley 100 de 1993 y sugieren un deterioro en el control de los riesgos de morir que pudo haber comenzado ya alrededor de 1990, período que coincide con la apertura económica, la crisis financiera del Sistema Nacional de Salud, el desmonte del sistema público estatal, la incertidumbre administrativa generada en las instituciones del Estado por la descentralización y el nuevo sistema de control de los determinantes ambientales. Llama la atención que, entre las mujeres, las causas evitables en su conjunto vienen disminuyendo sin variaciones significativas desde el principio del período; mientras la mortalidad general, que incluye causas evitables y no evitables, muestra una desaceleración desde 1990; esta disociación entre la MG y la ME podrían reflejar la influencia de nuevos riesgos y causas de morir no reconocidos como evitables por la clasificación de ME utilizada en el estudio. Al patrón 3 se ajusta posteriormente la ME por medidas preventivas, analizadas en su conjunto, cuya reducción se desacelera desde 1996, primero en mujeres (1996) y luego en hombres (1997); esta desaceleración sugiere un deterioro en el control de las acciones preventivas que pudo darse desde el año siguiente a la implantación de la Ley 100. Al final de la serie (2000) se observa una desaceleración significativa de la mortalidad no evitable en ambos sexos que no es detectable cuando se analizan hombres y mujeres por separado.

- Patrón 4. Causas de muerte que venían disminuyendo y viran hacia el aumento a mediados de 1990. El cambio en la tasa de variación de este grupo de causas es más marcado que en la categoría anterior, y sugiere un mayor deterioro. Es el caso del descenso en las muertes por tos ferina y por dengue entre los hombres que van hacia el aumento alrededor de 1993, la desnutrición infantil en niños y niñas (1995), la malaria en hombres y mujeres (1995). Estos cambios coinciden con el desmonte del SNS y la implantación del SGSSS. Los 
virajes al aumento en este grupo de causas podrían reflejar, entre otros factores, un deterioro en la efectividad del sistema de servicios para controlar los riesgos de morir. Es posible que el deterioro en algunos de estos indicadores, como es el caso del aumento en la mortalidad por desnutrición infantil, obedezca también a un incremento en el riesgo generado por los efectos de la política económica aplicada en la década de 1990; sin embargo el diseño del estudio no permite aclarar esta relación. En 1996 vira también hacia el aumento el grupo de otras causas no clasificables en la población general y a expensas de las mujeres.

- Patrón 5. Causas que venían en aumento desde el inicio de la serie y se deterioran aún más, como es el caso de las muertes por dengue en la población de ambos sexos que aceleran su tendencia al aumento en 1995. El cambio observado podría obedecer al efecto de un aumento en los riesgos, a un deterioro en el sistema de control o al efecto combinado de ambos, sin que se pueda precisar la naturaleza de la variación. Este análisis se debe hacercon cuidado porque las tasas mortalidad por dengue durante el primer período fueron más o menos bajas y la tasa de variación no fue significativamente distinta de cero, lo que implica que no puede descartarse un descenso en las muertes por dengue hasta 1992; en cualquier caso, los resultados sugieren que la mortalidad por esta causa se deterioró en las inmediaciones de 1993.

- Patrón 6. Causas que se inicialmente viran al deterioro y luego se recuperan. Es el caso de las muertes por desnutrición en todas las edades que van hacia el aumento en 1995, tanto en hombres como en mujeres, para disminuir de nuevo a partir de 2000 aunque las tasas ajustadas que se observaron al final de la serie son mayores a las de 1985. La mortalidad por causas obstétricas venía en descenso desde el comienzo de la serie hasta 1996; desde 1997 se observa un cambio significativo al aumento que se prolonga hasta 2000 , para disminuir de nuevo al año siguiente. La mortalidad por causas perinatales que disminuía desde el comienzo de la serie, se deterioró entre 1998 y 2000 y se desacelera luego al final de la serie. El incremento observado en las muertes obstétricas y perinatales podría obedecer parcialmente al mejoramiento en el sistema de clasificación de muertes hospitalarias ocurrido en 1997 como lo han descrito otros estudios ${ }^{19}$; la mortalidad hospitalaria materna y la perinatal pudo haber sido sensible de manera notable al mejoramiento en la calidad de los registros y la estructura proporcional de la mortalidad entre 1998 y 2002, muestra una reducción progresiva en la proporción anual de MCM que es compatible con esta interpretación. Sin embargo, el descenso en la proporción de MCM se acompaña también de un aumento gradual en la proporción de MOC, lo que sugiere una relación de competencia entre estas dos categorías y un desplazamiento de causas mal definidas al grupo de MOC. Más difícil aún es admitir que la mortalidad por causas obstétricas y perinatales obedezca a un aumento en la cobertura de los registros pues ni la MG ni la ME, cuya frecuencia en la población es mucho mayor, presentan aumentos significativos en las inmediaciones de 1998 que corroboren esta hipótesis; por otra parte, algunos estudios sugieren que la cobertura del registro de mortalidad sigue alta en las regiones más pobres del país ${ }^{20}$. Adicionalmente, en el caso de la mortalidad por causas obstétricas, el cambio al aumento se insinúa antes de las reformas en el sistema de registros. La mortalidad por cáncer del cuello uterino que descendía hasta 1991; entre 1992 y 1996 este indicador se deteriora de forma marcada por cinco años, para disminuir de nuevo desde 1997 aunque a un ritmo menor que el de la primera fase. La reducción de la mortalidad por cáncer de cérvix desde 1997 y de la mortalidad por causas obstétricas y perinatales a partir de 2001, pueden reflejar los esfuerzos promovidos en esta época por el SGSSS, en cuyo caso sería necesario admitir no sólo las bondades de una política decidida de control sectorial en el campo de la salud sexual y reproductiva, sino también los fallos del sistema durante el período inmediatamente anterior. De todos modos, las tasas de muertes obstétricas y por cáncer cérvico-uterino observadas al final de la serie seguían mostrando valores similares a los de 1991.

Patrón 7. Causas que venían en aumento, se reducen y luego se agravan. Las ME por medidas mixtas, especialmente a expensas de las causas externas en los hombres, aumentaron entre el principio de la serie y 1992, cuando los homicidios hicieron su pico máximo impulsados por el narcotráfico y la violencia política; entre 1993 y 1997 mostraron un descenso, para recrudecer desde 1998 hasta el final de la serie; este grupo de defunciones pudiera reflejar el impacto limitado de las políticas sociales sobre los conflictos sociales y las formas de violencia de este período, más que el efecto de las reformas sanitarias. 
Gómez-Arias ${ }^{8}$ analizó la ME en Colombia con datos disponibles hasta 2001; pero en el momento de hacer el estudio los análisis de joinpoint no disponían de pruebas estandarizadas para evaluar la significancia de la tasa de variación de cada segmento. Los análisis de joinpoint son reproducibles en una misma serie, pero varían cuando se incorporan datos nuevos. Sin embargo, casi todos los resultados del presente trabajo confirman los hallazgos de la investigación anterior. Los patrones de tendencia encontrados por ambos estudios fueron similares, con las siguientes excepciones: En la investigación previa ${ }^{8}$ no se detectaban la desaceleración en la mortalidad por tétanos entre los hombres que aparece en este estudio, ni la recuperación en la mortalidad por desnutrición que ocurre al final de la serie, pero en cambio aparecía un deterioro en la mortalidad por tos ferina en mujeres que no se encontró en la presente serie.

\section{CONCLUSIÓN}

En Colombia, las reformas implantadas por el DANE entre 1997 y 1998 pudieron haber mejorado la calidad de los registros de mortalidad; sin embargo, no se observaron variaciones significativas en la tendencia de la $\mathrm{MG}$ y la ME que reflejen un aumento concomitante en la cobertura, con excepción de algunas de las muertes obstétricas y perinatales cuyo registro pudo aumentar en las inmediaciones de 1999 a expensas de casos que antes eran clasificados en otras categorías. Si se considera que las proyecciones censales sobreestiman la población real y que la omisión del registro pudiera seguir elevada en algunas regiones del país, es necesario pensar que las tasas reales de la mortalidad son mayores que las observadas en este estudio y que la subestimación puede ser mayor al final de la serie. La MG disminuyó en ambos sexos durante todo el período, sin variaciones significativas en la tendencia que se puedan relacionar de forma específica con el impacto favorable de las reformas sanitarias de los últimos años. Este patrón de reducción de la MG varía por sexo. Entre los hombres la MG muestra una tendencia irregular hacia la reducción y su riesgo de morir fue mayor que el de las mujeres, especialmente por efecto de la violencia que pesa mucho en el perfil epidemiológico de Colombia. Entre las mujeres la MG disminuyó también, pero muestra desde 1990 un aumento en los riesgos de morir.
La tendencia de la ME, considerada globalmente, tampoco mostró disminuciones significativas que reflejen el efecto favorable de las reformas sanitarias aplicadas en 1990 y 1993; en cambio, varias causas de ME aumentan desde entonces pues muestran patrones de tendencia distintos que sugieren un deterioro en el control de los determinantes de la muerte. El diseño del estudio y sus fundamentos epistemológicos no permiten afirmar que el mejoramiento o deterioro observado en los niveles de mortalidad ocurran de modo exclusivo como consecuencia de los cambios en la política sanitaria. Es posible afirmar, sin embargo, que: a) se observa un marcado deterioro en el control de causas controlables de muerte que venían disminuyendo en Colombia antes de 1990, y cuya intervención constituye una responsabilidad directa del sistema de salud, y b) que los cambios en las políticas sociales y sanitarias de los últimos años no se han reflejado en un mejoramiento en la tendencia de muertes que son evitables, a pesar de que el gasto en salud se incrementó de manera nostable durante el mismo período.

Conflicto de intereses. Los autores declaran que no hay conflicto de intereses en el presente manuscrito.

\section{AGRADECIMIENTOS}

Al Programa de Subvenciones para la Investigación de la OPS y a las universidades de Alicante y Antioquia por su apoyo al presente estudio.

\section{REFERENCIAS}

1. World Health Organization. The World Health Report 2000. Geneve: WHO; 2001.

2. Thomson H, Hoskins R, Petticrew M. Evaluating the health effects of social interventions. BMJ 2004; 328: 282-5.

3. Nolte E, McKee M. Does health care save lives? Avoidable mortality revisited. London: The Nuffield Trust; 2004.

4. Rutstein D, Beriberi W, Charmers T, Child CG, 3rd, Fishman AP, Perrin EB. Measuring the quality of medical care. NEngl J Med. 1976; 294: 582-8.

5. Holland WW, Fitzgerald AP, Hildrey SJ, Phillips SJ. Heaven can wait. J Public Health Med. 1994; 16: 321-30.

6. Uemura K, Pisa Z. Recent trends in cardiovascular diseases mortality in 27 industrialized countries. World Health Stat $Q$. 1985; 38: 142-62.

7. Holland WW (ed.). European Community Atlas of «Avoidable Death».2nd ed. N ${ }^{\circ}$ 6. Oxford: Oxford Medical Publications; 1991.

8. Gómez-Arias R. La mortalidad evitable como indicador de 


\section{Colombia Médica}

desempeño de la política sanitaria. Colombia 1985-2001. Medellín: Editorial Universidad de Antioquia; 2008.

9. Hernández M. El derecho a la salud en Colombia: obstáculos estructurales para su realización. Rev Salud Publica. 2000; 2: 121-44

10. Bossert T, Chawla M, Bowser D, Beauvais J, Giedion U, Arbeláez JJ, et al. Applied research on decentralization of health. Systems in Latin America: Colombia case study. 32 ed. Boston: Harvard School of Public Health; 2002.

11. Barón-Leguizamón G. Gasto nacional en salud de Colombia 1993-2003: composición y tendencias. Rev Salud Publica. 2007; 9:167-79.

12. Organización Panamericana de la Salud, Organización Mundial de la Salud. Clasificación estadística internacional de enfermedades y problemas relacionados con la salud. Décima revisión. Publicación Científica $\mathrm{N}^{\circ}$ 554. Washington: OPS/ OMS; 2003.

13. Colombia, Departamento Administrativo Nacional de Estadísticas. Calidad de los datos: Censo 2005. Conciliación Censal 1985-2005. Bogotá: DANE; 2007.

14. Taucher E. Mortality in Chile, 1955-1975: trends and causes. Notas Población 1978; 6:113-42.
Vol. 40 N 4, 2009 (Octubre-Diciembre)

15. Gómez-Arias RD. La mortalidad evitable como indicador de impacto de la reforma sanitaria. Colombia 1985-2001. Rev Facul Nal Salud Publica. 2008; 26: 58-71.

16. Kim H-J, Fay M, Feuer E, Midthune D. Permutation tests for joinpoint regression with applications to cancer rates. Stat Med. 2000; 19: 335-51.

17. Cayuela A, Rodríguez-Domínguez S, López-Campos L, Otero-Candelera R, Rodríguez-Matutes C. Joinpoint regression analysis of lung cancer in Andalucía, 1975-2000. Ann Oncol. 2004; 15: 793-6.

18. García CA. Causas de muerte como indicadores indirectos de necesidades en salud: Una interpretación sociodemográfica. Los casos de la frontera norte y de Morelos 1980-1990. México: Centro Regional de Investigaciones Multidisciplinarias, CRIM UNAM; 2002.

19. RuizM. La mortalidadmaterna en Colombia 1998-2001 ¿Cuánto ha mejorado su estimación? Bogotá: Día Mundial de la Población; 2004.

20. Colombia, Departamento Nacional de Planeación. Los municipios colombianos hacia los objetivos de desarrollo delmilenio: Salud, educación y reducción de la pobreza. Bogotá: DNP; 2006. 